\title{
Quantitative Structure-Activity Relationship Model for the Fetal- Maternal Blood Concentration Ratio of Chemicals in Humans
}

\author{
Tomoyuki Takaku, ${ }^{* a}$ Hirohisa Nagahori, ${ }^{a}$ Yoshihisa Sogame, ${ }^{a}$ and Tatsuya Takagi ${ }^{b, c}$ \\ ${ }^{a}$ Environmental Health Science Laboratory, Sumitomo Chemical Co., Ltd.; 3-1-98 Kasugade-Naka, Konohana-ku, \\ Osaka 554-8558, Japan: ${ }^{b}$ Graduate School of Pharmaceutical Sciences, Osaka University; 1-6 Yamadaoka, Suita, \\ Osaka 565-0871, Japan: and ${ }^{c}$ Institute for Microbial Diseases, Osaka University; 3-1 Yamadaoka, Suita, Osaka \\ 565-0871, Japan.
}

Received December 25, 2014; accepted March 9, 2015

A quantitative structure-activity relationship (QSAR) model of the fetal-maternal blood concentration ratio (F/M ratio) of chemicals was developed to predict the placental transfer in humans. Data on $\mathrm{F} / \mathrm{M}$ ratio of 55 compounds found in the literature were separated into training (75\%, 41 compounds) and testing sets (25\%, 14 compounds). The training sets were then subjected to multiple linear regression analysis using the descriptors of molecular weight (MW), topological polar surface area (TopoPSA), and maximum E-state of hydrogen atom (Hmax). Multiple linear regression analysis and a cross-validation showed a relatively high adjusted coefficient of determination $\left(R_{\mathrm{a}}^{2}\right)(0.73)$ and cross-validated coefficient of determination $\left(Q^{2}\right)(0.71)$, after removing three outliers. In the external validation, $R^{2}$ for external validation $\left(R_{\text {pred }}^{2}\right)$ was calculated to be 0.51. These results suggested that the QSAR model developed in this study can be considered reliable in terms of its robustness and predictive performance. Since it is difficult to examine the $\mathbf{F} / \mathrm{M}$ ratio in humans experimentally, this QSAR model for prediction of the placental transfer of chemicals in humans could be useful in risk assessment of chemicals in humans.

Key words placental transfer; human; quantitative structure-activity relationship; fetal-maternal blood concentration ratio

The placental transfer of chemicals is crucial for toxicity assessment of fetus, because chemicals that do not penetrate the placenta are considered safe for fetus. ${ }^{1)}$ Therefore, investigations of many drugs that are transferred from mother to fetus through the placenta have been performed.

Since it is difficult to measure the placental transfer of chemicals in humans ethically, in vitro and ex vivo methods have been developed and used for the evaluation of human placental transfer. ${ }^{2)}$ In in vitro methods, cell lines from human placenta, such as BeWo, JAr, and JEG cells, have been commonly used as models of the placental barrier. ${ }^{3)}$ In ex vivo methods, the human placenta is perfused to investigate the transfer of chemicals from maternal to fetal compartment. Although this system has been validated in many studies, and therefore this system is considered reliable, there are difficulties in conducting this evaluation method. ${ }^{2,4)}$

Quantitative structure-activity relationship (QSAR) models are useful tools for predicting the biological activity of chemicals. Descriptors of chemicals such as molecular weight and $\log P$, are needed to develop QSAR prediction, and those are determined by experiments, calculation, or predicted using software. In the field of pharmacokinetics, QSAR models have been developed and reported in areas such as oral bioavailability, Caco-2 permeability, and metabolism. ${ }^{5-7)}$

QSAR models for predicting the placental transfer of chemicals using ex vivo data have already been reported. ${ }^{8,9)}$ However, to our knowledge, this is the first report that indicates prediction of the fetal-maternal blood concentration ratio (F/M ratio) of chemicals using in vivo data. To predict the F/M ratio of chemicals in humans, we developed a QSAR model using the data of umbilical cord/maternal blood concentration ratio, derived from information found in the literature. Our model could be useful in fetal toxicity assessment.

\section{MATERIALS AND METHODS}

Data Sets The F/M ratio of 55 compounds was used as data sets. ${ }^{10-25)}$ Although the $\mathrm{F} / \mathrm{M}$ ratio of many compounds was directly cited from literature sources such as clinical studies, the ratio for some compounds (carnitine, chlordane, chlorpyrifos, dichlorodiphenyldichloroethylene (DDE), diazinon, dicloran, hexachlorobenzene (HCB) heptachlor epoxide, nonachlor, oxychlordane, and phthalimide) was calculated based on the concentration in umbilical cord and maternal blood.

Descriptors PaDEL-descriptor (version2.18) is a free software application developed at the National University of Singapore, which can calculate 770 2D descriptors. ${ }^{26)}$ Regarding the membrane penetration, the importance of molecular size and lipophilicity of compounds has been reported, and thus for the analysis, the descriptors of molecular weight (MW) and $X \log P$ were selected. ${ }^{8,9)}$ In addition, the descriptors of sum of the atomic polarizabilities (Apol), autocorrelation of a topological structure (ATS) descriptor, weighted by charges (ATSc1, ATSc2, ATSc3, ATSc4, ATSc5), ATS descriptor, weighted by polarizability (ATSp1, ATSp2, ATSp3, ATSp4, ATSp5), sum of the absolute value of the difference between atomic polarizabilities of all bonded atoms in the molecule (Bpol), maximum E-state of hydrogen atom (Hmax), maximum E-state (Gmax), minimum E-state of hydrogen atom (Hmin), minimum E-state (Gmin), Global topological charge index (GlobalTopoChargeIndex), combined dipolarity/ polarizability (MLFER_S), and topological polar surface area (TopoPSA) were chosen because those parameters are in- 
volved in the polarity of chemicals. ${ }^{27-31)}$ Descriptors for which correlation coefficients for other descriptors of the training sets were more than 0.7 were eliminated to avoid the multicollinearity. Finally, the descriptors of ATSc3, ATSc4, ATSp1, Hmax, Gmax, Hmin, GlobalTopoChargeIndex, TopoPSA, MW, and $X \log P$ were used to develop the QSAR model.

Development of in Silico Prediction Model The data sets were separated into training (75\%) and testing sets $(25 \%)$ using random number generation. Training sets were subjected to multiple linear regression analysis, using $\mathrm{R}$ programming language (version 3.1.0). The model with the lowest Akaike Information Criterion (AIC) was generated by a backward selection procedure using 10 descriptors. ${ }^{32)}$ Subsequently, $p$ values $(0.05)$ of significance tests for the regression coefficients were referenced to select descriptors. As the cross-validation of the model, Leave-one-out Cross-Validation (LOO-CV) was

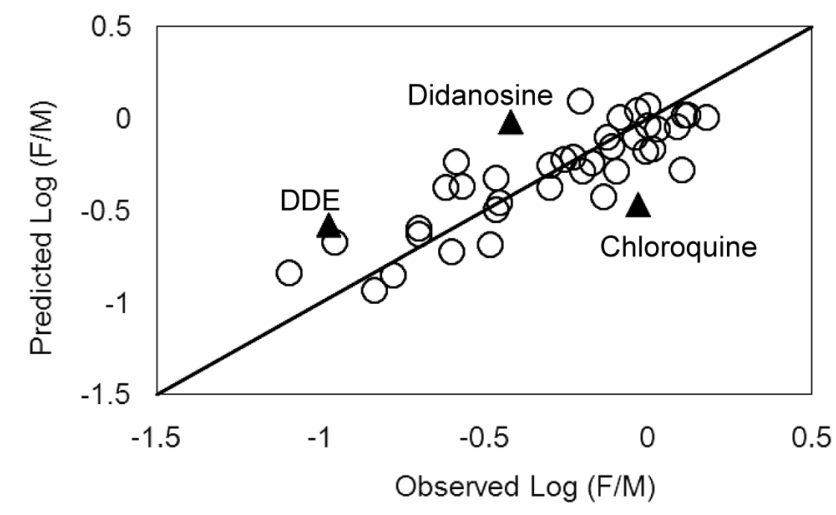

Fig. 1. A Plot of Observed vs. Predicted $\log (\mathrm{F} / \mathrm{M})$ Ratio of Training Sets

Solid triangles indicate outliers.

Table 1. Names, Descriptors, and Observed and Predicted $\log (\mathrm{F} / \mathrm{M})$ Ratio of Training Sets

\begin{tabular}{|c|c|c|c|c|c|c|}
\hline Name & Hmax & TopoPSA & MW & $\begin{array}{l}\text { Observed } \\
\log (\mathrm{F} / \mathrm{M})\end{array}$ & $\begin{array}{l}\text { Predicted } \\
\log (\mathrm{F} / \mathrm{M})\end{array}$ & Reference \\
\hline 17-Hydroxyprogesterone caproate & 0.582 & 60.4 & 428 & -0.70 & -0.63 & 10 \\
\hline Abacavir & 0.689 & 101.9 & 286 & 0.01 & -0.16 & 11 \\
\hline Acebutolol & 0.753 & 87.7 & 336 & -0.10 & -0.29 & 12 \\
\hline Buprenorphine & 1.250 & 62.2 & 467 & -0.46 & -0.46 & 15 \\
\hline Carnitine & 0.670 & 60.4 & 161 & 0.11 & 0.02 & 16 \\
\hline Cefoperazone & 1.015 & 264.3 & 648 & -0.46 & -0.49 & 17 \\
\hline Chlordane & 0.230 & 0.0 & 406 & -0.78 & -0.85 & 18 \\
\hline Chloroquine & 0.544 & 28.2 & 319 & -0.03 & -0.47 & 17 \\
\hline Chlorpyrifos & 1.154 & 82.5 & 349 & -0.01 & -0.18 & 19 \\
\hline Clindamycin & 0.814 & 127.6 & 424 & -0.30 & -0.37 & 17 \\
\hline Clonazepam & 0.841 & 84.6 & 315 & -0.23 & -0.21 & 17 \\
\hline DDE & 0.407 & 0.0 & 316 & -0.98 & -0.58 & 18 \\
\hline Diazepam & 0.791 & 32.7 & 284 & 0.10 & -0.28 & 14 \\
\hline Dicloran & 0.674 & 69.2 & 206 & 0.03 & -0.06 & 19 \\
\hline Didanosine & 0.849 & 88.7 & 236 & -0.42 & -0.02 & 11 \\
\hline Ethabutol & 0.577 & 64.5 & 204 & -0.12 & -0.10 & 17 \\
\hline Etidocaine & 0.637 & 32.3 & 276 & -0.47 & -0.32 & 21 \\
\hline Flecainide & 1.391 & 59.6 & 414 & -0.20 & -0.29 & 17 \\
\hline Flupenthixol & 1.339 & 52.0 & 434 & -0.62 & -0.37 & 17 \\
\hline $\mathrm{HCB}$ & 0.150 & 0.0 & 282 & -0.70 & -0.60 & 18 \\
\hline Heptachlor epoxide & 0.468 & 12.5 & 386 & -0.48 & -0.69 & 18 \\
\hline Indinavir & 0.795 & 118.0 & 613 & -1.10 & -0.84 & 14 \\
\hline Isoniazid & 0.675 & 68.0 & 137 & -0.21 & 0.10 & 17 \\
\hline Lamivudine & 0.819 & 113.5 & 229 & -0.03 & 0.05 & 11 \\
\hline Lidocaine & 0.637 & 32.3 & 234 & -0.26 & -0.23 & 17 \\
\hline Metronidazole & 0.735 & 81.2 & 171 & 0.00 & 0.07 & 17 \\
\hline Mifepristone & 0.595 & 40.5 & 429 & -0.96 & -0.67 & 17 \\
\hline Naloxone & 0.890 & 70.0 & 327 & -0.30 & -0.26 & 17 \\
\hline Nelfinavir & 0.768 & 127.2 & 567 & -0.60 & -0.72 & 23 \\
\hline Nevirapine & 0.624 & 58.1 & 266 & -0.17 & -0.24 & 22 \\
\hline Nifedipine & 1.039 & 107.8 & 346 & -0.11 & -0.15 & 24 \\
\hline Nonachlor & 0.228 & 0.0 & 440 & -0.84 & -0.93 & 18 \\
\hline Phthalimide & 0.635 & 46.2 & 147 & -0.09 & 0.01 & 19 \\
\hline Piperacillin & 1.052 & 181.7 & 517 & -0.57 & -0.37 & 17 \\
\hline Propranolol & 0.707 & 41.5 & 259 & -0.59 & -0.23 & 14 \\
\hline Remifentanil & 0.701 & 76.2 & 376 & -0.14 & -0.43 & 25 \\
\hline Sotalol & 1.019 & 86.8 & 272 & 0.00 & -0.04 & 12 \\
\hline Stavudine & 0.931 & 78.9 & 224 & 0.12 & 0.02 & 11 \\
\hline Ticarcillin & 0.980 & 177.6 & 384 & -0.04 & -0.10 & 17 \\
\hline Valproic acid & 0.669 & 37.3 & 144 & 0.18 & 0.01 & 14 \\
\hline Zidovudine & 0.945 & 91.2 & 267 & 0.09 & -0.05 & 11 \\
\hline
\end{tabular}


conducted to evaluate the predictive performance, and the cross-validated coefficient of determination $\left(Q^{2}\right)$ was used as the indicator. ${ }^{33)}$ In addition, an external validation was made, which is considered the best method of evaluation of the model, and $R^{2}$ for external validation $\left(R_{\text {pred }}^{2}\right)$ was used as the indicator. $^{33)}$

\section{RESULTS AND DISCUSSION}

Multiple Linear Regression Analysis By the AIC backward selection procedure and the selection based on $p$ values (0.05) of significance tests for the regression coefficients, 3 descriptors (MW, TopoPSA, and Hmax) were selected to develop the model of training sets (41 compounds). MW and TopoPSA are the descriptors of molecular size and polar surface area of compounds, respectively, and involved in the membrane penetration of placenta. ${ }^{89}$ Votano et al. reported that Hmax is the maximum hydrogen atom E-state value in a compound, and correlates with charge. ${ }^{34)}$ For example, the hydrogen atoms of $\mathrm{OH}, \mathrm{NH}_{2}$, and $\mathrm{COOH}$ groups tend to be Hmax. However, the involvement of Hmax for the placental transfer has not been reported.

Multiple linear regression analysis of training sets and a cross-validation were conducted as described below (Eq. 1) (Fig. 1, Table 1). Values in parentheses indicate the 95\% confidence limits.

$$
\begin{aligned}
\log (\mathrm{F} / \mathrm{M})= & -0.00231( \pm 0.000581) \mathrm{MW} \\
& +0.00233( \pm 0.00160) \mathrm{TopoPSA} \\
& +0.382( \pm 0.285) \mathrm{Hmax}-0.00363 \\
n=41, R_{\mathrm{a}}^{2}= & 0.64, Q^{2}=0.63
\end{aligned}
$$

Because the absolute values of the standardized residuals of three compounds (chloroquine, DDE, and didanosine) were more than two, these compounds were considered outliers. After removing these three outliers, multiple linear regression analysis and a cross-validation were conducted again (Eq. 2). Values in parentheses indicate the $95 \%$ confidence limits.

$$
\begin{aligned}
\log (\mathrm{F} / \mathrm{M})= & -0.00238( \pm 0.00050) \mathrm{MW} \\
& +0.00238( \pm 0.00139) \mathrm{TopoPSA} \\
& +0.380( \pm 0.245) \mathrm{Hmax}+0.0283 \\
n=38, R_{\mathrm{a}}^{2}= & 0.73, Q^{2}=0.71
\end{aligned}
$$

To clarify the importance of the descriptors used in multiple linear regression analysis, the standard partial regression coefficients were calculated, and those values for MW, TopoPSA, and Hmax were $-0.922,0.376$, and 0.314 , respectively. These results suggest that the MW descriptor is more important for the $\mathrm{F} / \mathrm{M}$ ratio in humans than the polarity descriptors.

The MW descriptor was negative-valued in our prediction model, in common with an ex vivo model. ${ }^{9}{ }^{9}$ This means that for decreasing values of $\mathrm{MW}$, the $\mathrm{F} / \mathrm{M}$ ratio increases. Since small compounds penetrate the placental membrane more easily than large compounds, the negative value of MW seems reasonable in terms of the F/M ratio. ${ }^{9)}$ On the other hand, the polarity descriptors (TopoPSA and Hmax) were positive-valued in the model, suggesting that when TopoPSA and Hmax decrease the $\mathrm{F} / \mathrm{M}$ ratio also decreases. It should be noted that in the ex vivo model for prediction of the $\mathrm{F} / \mathrm{M}$ ratio, those descriptors were negative-valued, different from our model.

\begin{tabular}{|c|c|c|c|c|c|c|}
\hline Name & $\operatorname{Hmax}$ & TopoPSA & MW & $\begin{array}{l}\text { Observed } \\
\log (\mathrm{F} / \mathrm{M})\end{array}$ & $\begin{array}{l}\text { Predicted } \\
\log (\mathrm{F} / \mathrm{M})\end{array}$ & Reference \\
\hline Atazanavir & 0.910 & 171.2 & 704 & -0.89 & -0.90 & 13 \\
\hline Bupivacaine & 0.637 & 32.3 & 288 & -0.52 & -0.34 & 14 \\
\hline Clonidine & 0.492 & 36.4 & 229 & -0.05 & -0.24 & 17 \\
\hline Diazinon & 1.173 & 95.4 & 304 & 0.00 & -0.02 & 19 \\
\hline Disopyramide & 0.662 & 59.2 & 339 & -0.59 & -0.39 & 17 \\
\hline Duloxetine & 0.701 & 49.5 & 297 & -0.92 & -0.29 & 20 \\
\hline Indomethacin & 0.820 & 68.5 & 357 & -0.01 & -0.35 & 17 \\
\hline Lopinavir & 0.902 & 120.0 & 628 & -0.62 & -0.84 & 22 \\
\hline Metoprolol & 0.691 & 50.7 & 267 & 0.00 & -0.22 & 12 \\
\hline Midazolam & 0.785 & 29.7 & 325 & -0.13 & -0.38 & 17 \\
\hline Norbuprenorphine & 0.768 & 71.0 & 413 & -0.31 & -0.49 & 15 \\
\hline Oxprenolol & 0.727 & 50.7 & 265 & -0.43 & -0.21 & 12 \\
\hline Oxychlordane & 0.465 & 12.5 & 420 & -1.02 & -0.76 & 18 \\
\hline Procainamide & 0.549 & 58.4 & 235 & 0.04 & -0.18 & 17 \\
\hline
\end{tabular}
In the ex vivo model, the $\mathrm{F} / \mathrm{M}$ ratio is decided based only on the effect of membrane penetration, because a buffer is used

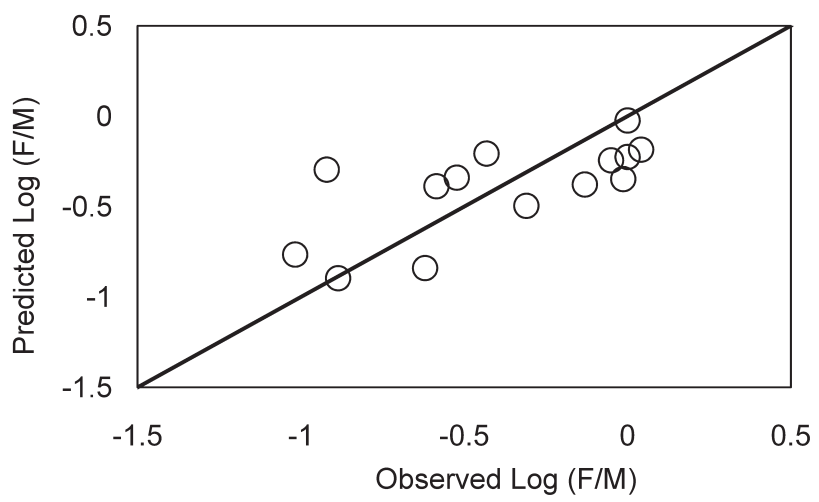

Fig. 2. The Plot of Observed vs. Predicted $\log (\mathrm{F} / \mathrm{M})$ Ratio of Testing Sets

Solid triangles indicate outliers.

Table 2. Names, Descriptors, and Observed and Predicted $\log (\mathrm{F} / \mathrm{M})$ Ratio of Testing Sets 
in the ex vivo experimental system instead of umbilical cord and maternal blood, and the impact on the interaction of compounds with blood lipids is ignored. Thus, under the ex vivo conditions, compounds of low polarity would be more easily transported across the placental membrane than those of high polarity, because of the high lipid content in the membrane, leading to the negative values of the polarity descriptors.

In contrast, the determination of the in vivo $\mathrm{F} / \mathrm{M}$ ratio was based on the influence of both membrane penetration and interaction with blood lipids. Because maternal blood contains several times higher lipid content than newborn blood, compounds of low polarity would more easily exist in the maternal blood. ${ }^{35)}$ In addition, uptake transporters may be involved in the placental transfer of chemicals. ${ }^{36)}$ For example, it was reported that the placental transfer of valproic acid is mediated by monocarboxylic acid transporters (MCT). ${ }^{37)}$ In general, substrates of membrane transporters are considered hydrophilic. ${ }^{38)}$ Therefore, the positive values of the polarity descriptors (TopoPSA and Hmax) might be attributed to uptake transporters. These findings suggest that the F/M ratio of chemicals in vivo would be described on the basis of both membrane penetration and interaction with blood lipids.

External Validation Because the data sets were separated into training $(75 \%)$ and testing sets $(25 \%)$ using random number generation, external validation was conducted using testing sets (14 compounds), showing that $R_{\text {pred }}^{2}$ was 0.51 , which is considered an acceptable model ${ }^{33)}$ (Fig. 2, Table 2).

\section{CONCLUSION}

A QSAR model for prediction of the placental transfer in humans was developed using $\mathrm{F} / \mathrm{M}$ ratio of 55 compounds. The model for training sets (41 compounds) was developed using multiple linear regression analysis with three descriptors (MW, TopoPSA, and Hmax). The predictive ability of the model was confirmed by external validation (14 compounds). This QSAR model could be useful in risk assessment of placental transfer of chemicals to fetus, because it is difficult to determine the $\mathrm{F} / \mathrm{M}$ ratio of humans experimentally.

Conflict of Interest Tomoyuki Takaku, Hirohisa Nagahori, and Yoshihisa Sogame are employees of Sumitomo Chemical Co., Ltd.

\section{REFERENCES}

1) Gupta RC. Toxicology of the placenta. General, applied and systems toxicology. John Wiley \& Sons, Ltd. (2009).

2) Myren M, Mose T, Mathiesen L, Knudsen LE. The human placenta - an alternative for studying foetal exposure. Toxicol. In Vitro, 21, 1332-1340 (2007).

3) Vähäkangas K, Myllynen P. Experimental methods to study human transplacental exposure to genotoxic agents. Mutat. Res., 608, $129-135$ (2006).

4) Grafmüller S, Manser P, Krug HF, Wick P, von Mandach U. Determination of the transport rate of xenobiotics and nanomaterials across the placenta using the ex vivo human placental perfusion model. J. Vis. Exp., 76, e50401 (2013).

5) Andrews CW, Bennett L, Yu LX. Predicting human oral bioavailability of a compound: development of a novel quantitative structure-bioavailability relationship. Pharm. Res., 17, 639-644 (2000).

6) Yamashita F, Wanchana S, Hashida M. Quantitative structure/prop- erty relationship analysis of Caco-2 permeability using a genetic algorithm-based partial least squares method. J. Pharm. Sci., 91, 2230-2239 (2002).

7) Smith PA, Sorich MJ, McKinnon RA, Miners JO. Pharmacophore and quantitative structure-activity relationship modeling: complementary approaches for the rationalization and prediction of UDPglucuronosyltransferase 1A4 substrate selectivity. J. Med. Chem., 46, 1617-1626 (2003).

8) Hewitt M, Madden JC, Rowe PH, Cronin MT. Structure-based modelling in reproductive toxicology: (Q)SARs for the placental barrier. SAR QSAR Environ. Res., 18, 57-76 (2007).

9) Giaginis C, Zira A, Theocharis S, Tsantili-Kakoulidou A. Application of quantitative structure-activity relationships for modeling drug and chemical transport across the human placenta barrier: a multivariate data analysis approach. J. Appl. Toxicol., 29, 724-733 (2009).

10) Caritis SN, Sharma S, Venkataramanan R, Hankins GD, Miodovnik M, Hebert MF, Umans JG, Benedetti T, Mattison D, Zajicek A, Fischer D, Jackson A, Eunice Kennedy Shriver National Institute of Child Health and Human Development Obstetrical-Fetal Pharmacology Research Units Network. Pharmacology and placental transport of 17-hydroxyprogesterone caproate in singleton gestation. Am. J. Obstet. Gynecol., 207, 398.e1-398.e8 (2012).

11) Chappuy H, Tréluyer JM, Jullien V, Dimet J, Rey E, Fouché M, Firtion G, Pons G, Mandelbrot L. Maternal-fetal transfer and amniotic fluid accumulation of nucleoside analogue reverse transcriptase inhibitors in human immunodeficiency virus-infected pregnant women. Antimicrob. Agents Chemother., 48, 4332-4336 (2004).

12) Sioufi A, Hillion D, Lumbroso P, Wainer R, Olivier-Martin M, Schoeller JP, Colussi D, Leroux F, Mangoni P. Oxprenolol placental transfer, plasma concentrations in newborns and passage into breast milk. Br. J. Clin. Pharmacol., 18, 453-456 (1984).

13) Ripamonti D, Cattaneo D, Maggiolo F, Airoldi M, Frigerio L, Bertuletti P, Ruggeri M, Suter F. Atazanavir plus low-dose ritonavir in pregnancy: pharmacokinetics and placental transfer. $A I D S, 21$, 2409-2415 (2007).

14) Hutson JR. Prediction of placental drug transfer using the human placental perfusion model. J. Popul. Ther. Clin. Pharmacol., 18, e533-e543 (2011)

15) Gordon AL, Lopatko OV, Somogyi AA, Foster DJ, White JM. $(R)$ - and $(S)$-Methadone and buprenorphine concentration ratios in maternal and umbilical cord plasma following chronic maintenance dosing in pregnancy. Br. J. Clin. Pharmacol., 70, 895-902 (2010).

16) Winter SC, Linn LS, Helton E. Plasma carnitine concentrations in pregnancy, cord blood, and neonates and children. Clin. Chim. Acta, 243, 87-93 (1995).

17) Briggs GG, Freeman RK, Yaffe SJ. Drugs in pregnacy and lactation, 6th edition. Lippincott Williams \& Wilkins Publishers (2001).

18) Fukata H, Omori M, Osada H, Todaka E, Mori C. Necessity to measure PCBs and organochlorine pesticide concentrations in human umbilical cords for fetal exposure assessment. Environ. Health Perspect., 113, 297-303 (2005).

19) Whyatt RM, Barr DB, Camann DE, Kinney PL, Barr JR, Andrews HF, Hoepner LA, Garfinkel R, Hazi Y, Reyes A, Ramirez J, Cosme Y, Perera FP. Contemporary-use pesticides in personal air samples during pregnancy and blood samples at delivery among urban minority mothers and newborns. Environ. Health Perspect., 111, 749-756 (2003)

20) Boyce PM, Hackett LP, Ilett KF. Duloxetine transfer across the placenta during pregnancy and into milk during lactation. Arch. Women Ment. Health, 14, 169-172 (2011).

21) Morgan DJ, Cousins MJ, McQuillan D, Thomas J. Disposition and placental transfer of etidocaine in pregnancy. Eur. J. Clin. Pharmacol., 12, 359-365 (1977).

22) van Hoog S, Boer K, Nellen J, Scherpbier H, Godfried MH. Transplacental passage of nevirapine, nelfinavir and lopinavir. Neth. J. 
Med., 70, 102-103 (2012).

23) Hirt D, Urien S, Jullien V, Firtion G, Chappuy H, Rey E, Pons G, Mandelbrot L, Treluyer JM. Pharmacokinetic modelling of the placental transfer of nelfinavir and its M8 metabolite: a population study using 75 maternal-cord plasma samples. Br. J. Clin. Pharmacol., 64, 634-644 (2007).

24) Silberschmidt AL, Kühn-Velten WN, Juon AM, Zimmermann R, von Mandach U. Nifedipine concentration in maternal and umbilical cord blood after nifedipine gastrointestinal therapeutic system for tocolysis. BJOG, 115, 480-485 (2008).

25) Ngan Kee WD, Khaw KS, Ma KC, Wong AS, Lee BB, Ng FF. Maternal and neonatal effects of remifentanil at induction of general anesthesia for cesarean delivery: a randomized, double-blind, controlled trial. Anesthesiology, 104, 14-20 (2006).

26) Yap CW. PaDEL-descriptor: an open source software to calculate molecular descriptors and fingerprints. J. Comput. Chem., 32, 1466-1474 (2011).

27) Galvez J, Garcia R, Salabert MT, Soler R. Charge indexes. New topological descriptors. J. Chem. Inf. Comput. Sci., 34, 520-525 (1994).

28) Ertl P, Rohde B, Selzer P. Fast calculation of molecular polar surface area as a sum of fragment-based contributions and its application to the prediction of drug transport properties. J. Med. Chem., 43, 3714-3717 (2000).

29) Kier LB, Hall LH. An electrotopological-state index for atoms in molecules. Pharm. Res., 7, 801-807 (1990).

30) Platts JA, Butina D, Abraham MH, Hersey A. Estimation of mo- lecular linear free energy relation descriptors using a group contribution approach. J. Chem. Inf. Comput. Sci., 39, 835-845 (1999).

31) Moreau G, Broto P. The autocorrelation of a topological structure: A new molecular descriptor. Nouv. J. Chim., 4, 359-360 (1980).

32) Hughes AW, King ML. Model selection using AIC in the presence of one-sided information. J. Stat. Plan. Inference, 115, 397-411 (2003).

33) Pratim Roy P, Paul S, Mitra I, Roy K. On two novel parameters for validation of predictive QSAR models. Molecules, 14, 1660-1701 (2009).

34) Votano JR, Parham M, Hall LH, Kier LB, Oloff S, Tropsha A, Xie Q, Tong W. Three new consensus QSAR models for the prediction of Ames genotoxicity. Mutagenesis, 19, 365-377 (2004).

35) Peticca P, Raymond A, Gruslin A, Cousins M, Adetola E, Abujrad H, Mayne J, Ooi TC. Human serum PCSK9 is elevated at parturition in comparison to nonpregnant subjects while serum PCSK9 from umbilical cord blood is lower compared to maternal blood. ISRN Endocrinol., 2013, 341632 (2013).

36) Rubinchik-Stern M, Eyal S. Drug interactions at the human placenta: What is the evidence? Front. Pharmacol., 3, 126 (2012).

37) Utoguchi N, Audus KL. Carrier-mediated transport of valproic acid in BeWo cells, a human trophoblast cell line. Int. J. Pharm., 195, $115-124$ (2000)

38) Mishra NK, Chang J, Zhao PX. Prediction of membrane transport proteins and their substrate specificities using primary sequence information. PLoS ONE, 9, e100278 (2014). 